\title{
New Method for Determination of Surface Potential of Microscopic Particles by Second Harmonic Generation
}

\author{
Elsa C. Y. Yan, Yan Liu, and Kenneth B. Eisenthal* \\ Chemistry Department, Columbia University, New York, New York 10027
}

Received: February 27, 1998; In Final Form: June 2, 1998

\begin{abstract}
A new noninvasive method for determining the surface electrostatic potential and surface charge density of microscopic particles using second harmonic generation (SHG) is described. The surface electrostatic properties of $1.05 \mu \mathrm{m}$ polystyrene sulfate spheres in aqueous solution and that of $0.22 \mu \mathrm{m}$ oil droplets in aqueous emulsions are obtained. Comparisons of the surface potentials obtained from SHG with the zeta potential obtained from electrophoresis are in excellent agreement with theoretical predictions.
\end{abstract}

\section{Introduction}

Surface charge density $\sigma$ and surface potential $\Phi(0)$ are important characteristics of microscopic particles that have a significant effect on a number of chemical and physical properties. Among these are the populations and structural arrangements of neutral and ionic species in the surface region of the particle, the chemical reactions near the surface, the electrokinetic properties of the particle, and the stability of the particle suspension in the solution. This paper presents a new noninvasive method for determining the surface potential of microscopic particles utilizing surface second harmonic generation. This method has some advantages over traditional techniques.

The surface potential can be measured directly for conducting materials using an electrochemical cell. ${ }^{1}$ For nonconducting microparticles the conventional method to measure the surface potential is electrophoresis, in which the mobility $u$ of a charged particle is measured, and the zeta potential $\zeta$ is evaluated. ${ }^{2}$ However, $\zeta$ is not the potential at the interface, but the potential at the shear surface, which is the boundary that separates the mobile fluid and the immobilized layer of electrolyte solution that moves together with the particle. It is not precisely known where the shear surface is located. Assumptions about the distance between the shear layer and the interface have to be made in order to get the surface potential from $\xi .{ }^{3-5}$ Moreover, during the electrophoresis measurement the double layer of the microparticles is perturbed by both the motion of the microparticles and the electric field. In considering the motion of the particles, the viscosity in the double-layer region is an important parameter and once again assumptions have to be made to obtain the value of the viscosity inside the double layer. 6,7 Although the determination of surface potential from $\zeta$ has some difficulties, it remains of considerable value because it establishes a lower limit for the surface potential and serves as an empirical quantity to be correlated with the environment of microparticles in solution. There are other methods to investigate the surface potential of microscopic particles. These include the use of $\mathrm{pH}$ indicator molecules, ${ }^{8-10}$ fluorescent yield measurements, ${ }^{11}$ and recently atomic force microscopy. ${ }^{12}$ All of these methods are of considerable value but perturb the microscopic particles to be studied. Second harmonic and sum frequency methods are complementary to these traditional methods.
Second harmonic generation (SHG) is a surface specific technique for probing equilibrium and dynamic phenomena at interfaces. It has been used to study liquid/gas, liquid/liquid, liquid/solid, solid/gas, and solid/solid interfaces. ${ }^{13-16} \mathrm{SHG}$ is often described as electric dipole forbidden in centrosymmetric media, which can be understood by the following symmetry argument. In centrosymmetric bulk media, for every molecule oriented in one direction there is one oriented in the opposite direction. The nonlinear polarizations induced in these oppositely oriented molecules are of opposite phase with respect to each other and cancel. Therefore the net second-order polarization is zero, and there is no SHG generated from the very large population of molecules in the bulk medium. Unlike the molecules in the bulk liquid, the molecules at interfaces are oriented owing to the asymmetric forces acting on them. Thus symmetry is broken at the interface, which therefore makes it possible for the oriented molecules at the interfaces to generate a second-order polarization on exposure to an incident light wave. A coherent SHG is radiated from the interface, but not the bulk region, thus yielding a surface specific signal.

We now ask how SHG and SFG can be generated from the surface of centrosymmetric particles in an isotropic bulk liquid medium if these processes are electric dipole forbidden for centrosymmetric systems. The answer is that SHG and SFG are electric dipole forbidden in centrosymmetric systems provided the particle is centrosymmetric on length scales much less than the coherence length of the process. The coherence length, $L_{\mathrm{c}}$, of the process is given by the relation $\Delta k^{\circ} L_{\mathrm{c}} \sim \pi$, where $\Delta k=k_{2 \omega}-2 k_{\omega}$, and $k_{2 \omega}, k_{\omega}$ are the light propagation vectors for the SHG light and the fundamental light and are inversely proportional to their respective wavelengths in the medium. Although the microsized particle is centrosymmetric on a macroscopic scale, it is not centrosymmetric in any local region of the surface. There is no net cancellation from oppositely oriented molecules located on opposite sides of the microsized particle surface because the separation is comparable to the coherence length of the process. Thus the second harmonic generated at the opposing parts of the centrosymmetric particle's surface can add constructively to give a SHG signal. Strong SHG signals have been observed and reported for various microparticle systems. ${ }^{17-18}$

To describe how SHG is used to investigate the electrostatics of charged microscopic particles, we will briefly discuss how 


\section{SCHEME 1}

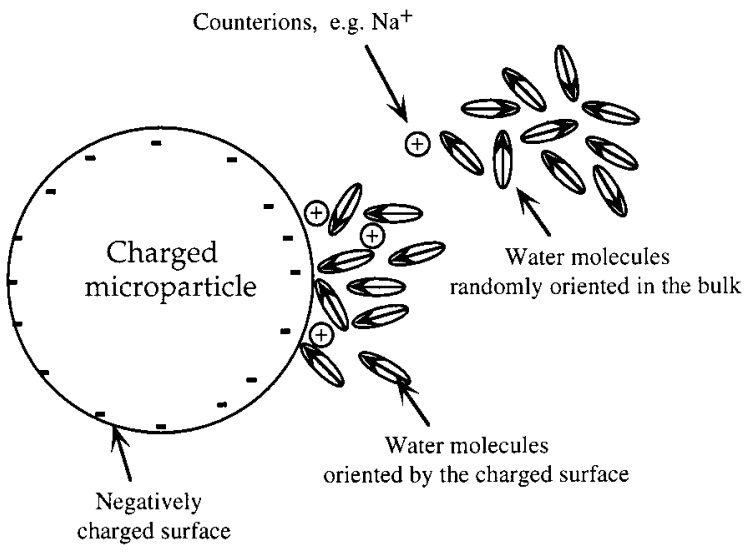

SHG has been used to study the electrostatic properties of the planar fused silica/water interface ${ }^{19}$ and that of charged monolayers at air/water interfaces. ${ }^{20,21}$ In these studies it was discovered that there were two contributions to the SHG signal from charged interfaces, namely, the second-order $\chi^{(2)}$ and thirdorder $\chi^{(3)}$ contributions. The $\chi^{(2)}$ contribution is from the molecules at the interface, which are oriented, and can therefore give an electric dipole allowed SHG signal. The $\chi^{(3)}$ contribution results from the electric field induced polarization of bulk water molecules by the charged interface. ${ }^{19-21}$ The centrosymmetry of the bulk water is broken owing to the static electric field, which extends into the bulk liquid, and is generated by the charges at the surface. The $\chi^{(3)}$ contribution contains information on the surface charge density and the surface potential of the charged interfaces.

We have combined the ideas of SHG studies on microparticle surfaces with studies on charged planar interfaces in order to probe charged microparticle/water interfaces. ${ }^{17,19-21}$ The experiments were carried out on two different types of microscopic particles. One system is a suspension of polystyrene sulfate (PSS) microspheres in water, for which the surface charge is due to sulfate groups. The other system is an $n$-tetradecane/ water emulsion consisting of $n$-tetradecane (oil) droplets in a water solution. The surface charge is due to the dodecyl sulfate $\left(\mathrm{C}_{12} \mathrm{H}_{25} \mathrm{SO}_{4}^{-}\right)$emulsifier. From this study, we were able to find the surface charge density and surface potential of the microspheres and the oil droplets without any perturbation or reliance on probe molecules. To our knowledge, this study presents the first determination of microparticle surface potentials by SHG.

\section{Method for Determination of Surface Potential of Charged Particles by Second Harmonic Generation}

1. SHG Signal Due to a Charged Interface. Because the radius of curvature of the microscopic particles is large, we can describe the electrostatics near the surface $(100 \AA)$ as if the interface was strictly planar. We can thus use the approach used in the SH studies of the charged planar interfaces, silica/ water, ${ }^{19}$ and charged surfactants at planar air/water interfaces. ${ }^{20-21}$

The second-order polarization $P_{2 \omega}{ }^{(2)}$ arising from the oriented molecules at the interface can be expressed as

$$
P_{2 \omega}^{(2)}=\chi^{(2)} E_{\omega} E_{\omega}
$$

where $E_{\omega}$ is the incident optical field at frequency $\omega$. A second contribution to the second harmonic signal is from the bulk solvent molecules polarized by the charged interface. The static electric field due to the surface charges can orient and induce a third-order polarization in the solvent; see Scheme 1. The third-order polarization induced at distance $z$ normal to the interface can be expressed as

$$
P_{2 \omega}^{(3)}(z)=\chi^{(3)} E_{\omega} E_{\omega} E_{0}(z)
$$

where $\chi^{(3)}$ is the third-order nonlinear susceptibility and $E_{0}$ is the static field due to the interface charges.

When we consider a charged surface in contact with water, the bulk water molecules experiencing the static electric field can contribute to $P_{2 \omega}{ }^{(3)}(z)$. The total third-order polarization, $P_{2 \omega}{ }^{(3)}$, is obtained by integrating eq 2 from the interface, $z=0$ to $z=\infty$, where the electric field decays to zero

$$
P_{2 \omega}{ }^{(3)}=\int_{0}^{\infty} d P_{2 \omega}{ }^{(3)}(z)=\int_{0}^{\infty} \chi^{(3)} E_{\omega} E_{\omega} E_{0}(z) \mathrm{d} z
$$

Carrying out the integration with the assumption that the density of water molecules does not change significantly from the vicinity of the interface to the bulk solution, we obtain

$$
\begin{aligned}
P_{2 \omega}{ }^{(3)} & =\chi^{(3)} E_{\omega} E_{\omega} \int_{0}^{\infty} E_{0}(z) \mathrm{d} z \\
& =-\chi^{(3)} E_{\omega} E_{\omega}[\Phi(\infty)-\Phi(0)] \\
& =\chi^{(3)} E_{\omega} E_{\omega} \Phi(0)
\end{aligned}
$$

where $\Phi(0)$ is the surface potential and $\Phi(\infty)$ is the potential at infinity, i.e., far into the bulk, which is set equal to zero.

Addition of the second- and third-order polarization gives the total polarization from a charged interface

$$
P_{2 \omega}=\chi^{(2)} E_{\omega} E_{\omega}+\chi^{(3)} E_{\omega} E_{\omega} \Phi(0)
$$

Since the second harmonic field $E_{\mathrm{SHG}}$ is proportional to $P_{2 \omega}$, $E_{\mathrm{SHG}}$ can be written as

$$
P_{2 \omega} \sim E_{\mathrm{SHG}}=A+B \Phi(0)
$$

where $A$ and $B$ contain the $\chi^{(2)}$ and $\chi^{(3)}$ contributions, respectively.

2. Effect of Electrolyte on the Second Harmonic Field, $\boldsymbol{E}_{\text {SHG }}$. When a charged interface contacts an electrolyte solution, the ions in the solution will interact with the charged interface and distribute themselves accordingly. The presence of counterions screens the static electric field due to the charged interface and decreases the magnitude of the field as the distance from the interface increases. The number of water molecules polarized by the field then decreases, which leads to a decrease of the magnitude of the $\chi^{(3)}$ contribution to $E_{\mathrm{SHG}}$. Therefore, $E_{\text {SHG }}$ changes with the bulk electrolyte concentration.

The Gouy-Chapman model of the electric double layer can be employed to describe the electric potential, $\Phi(0)$, of the charged microparticle interface. In this model, the PoissonBoltzmann equation is solved for a planar charged surface in contact with a symmetric electrolyte solution, e.g., univalent, $1: 1, \mathrm{KCl}$ or divalent, $2: 2, \mathrm{MgSO}_{4}$, with the assumption that all the ions are point charges. The expression is as follows

$$
\Phi(0)=\frac{2 k T}{Z e} \sinh ^{-1}\left(\sigma \sqrt{\frac{\pi}{2 \epsilon k T C}}\right)
$$

where $C$ is the total bulk electrolyte concentration, $k$ is the Boltzmann constant, $T$ is the temperature, $Z$ is the valence of 


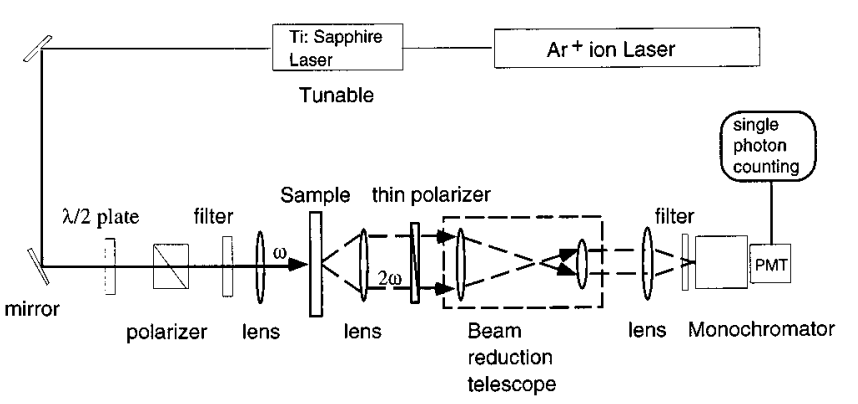

Figure 1. Experimental setup for second harmonic measurements.

the ions, $\sigma$ is the surface charge density, and $\epsilon$ is the dielectric constant of the bulk solution.

We can now obtain a model to fit the dependence of $E_{\text {SHG }}$ on electrolyte concentration $C$ by putting eq 7 into eq 6 . We obtain

$$
E_{\mathrm{SHG}}(C)=A+\mathrm{B}\left(\frac{2 k T}{Z e}\right) \sinh ^{-1}\left(\sigma \sqrt{\frac{\pi}{2 \epsilon k T C}}\right)
$$

in which $A, B$, and $\sigma$ are the fitting parameters. Once they are obtained from the fitting, $\Phi(0)$ can be found.

3. Hyper-Rayleigh Correction. In the experiment, the physical quantity measured was the total intensity of the light at frequency $2 \omega$. Although the measured SHG is from the microparticle surface, there can also be a signal at $2 \omega$ from the bulk liquid due to hyper-Rayleigh (HR) scattering. The HR scattering is an incoherent second-order process originating from the breaking of local symmetry due to the fluctuations in the orientation and density of solvent molecules. ${ }^{22-23}$ To obtain the SHG signal arising from the microparticle surface, we have to correct for the HR scattering. Water, being the bulk medium in the system, is the major source of the HR scattering. Apart from water, there are ions in the systems. The contribution from the ions in the solution was negligible on the basis of the experimental result that the HR intensity of pure water and electrolyte solution was the same within experimental error.

Since the hyper-Rayleigh scattering is an incoherent process, the total intensity, $I_{\text {total }}$, measured at frequency $2 \omega$ can be written as

$$
I_{\text {total }}=I_{\mathrm{SHG}}+I_{\mathrm{HR}}
$$

where $I_{\mathrm{SHG}}$ is the SHG intensity from the interface of the microparticles and $I_{\mathrm{HR}}$ is the intensity of hyper-Rayleigh scattering from water. Since the system is turbid owing to the presence of the PSS particles or the emulsion oil droplets, the contribution of $I_{\mathrm{HR}}$ is actually the measured hyper-Raleigh scattering intensity of neat water after correction for the attenuation by the PSS or the emulsion oil droplets. The HR from water without any microparticles was measured using the setup in Figure 1. The turbidities of the microparticle samples at $\omega$ and $2 \omega$ were measured with a UV-vis spectrometer. Considering the attenuation of $\omega$ and $2 \omega$ light, we can calculate the HR contribution from water in the microparticle suspension, $I_{\mathrm{HR}}$, by an integration along the path length. Therefore, $E_{\mathrm{SHG}}$ can be found by

$$
E_{\mathrm{SHG}}=\sqrt{I_{\mathrm{SHG}}}=\sqrt{\left(I_{\mathrm{total}}-I_{\mathrm{HR}}\right)}
$$

The $E_{\mathrm{SHG}}$ is obtained as a function of electrolyte concentration, which is then fitted to eq 8 .

\section{Experimental Section}

The setup for the second harmonic measurements is shown in Figure 1. A Ti:sapphire laser provides $1.4 \mathrm{~W}$ of $100 \mathrm{fs}$ pulses at $842 \mathrm{~nm}(\omega)$, at a repetition rate of $82 \mathrm{MHz}$. The fundamental light is gently focused into a $0.2 \mathrm{~cm}$ long quartz cell using a lens of focal length $7.5 \mathrm{~cm}$. Second harmonic photons at 421 $\mathrm{nm}(2 \omega)$ are collected in the transmitted direction by a condensing lens with an $f$ number of 1.1 and detected by a single-photon counting method. Filters and a monochromator separate the second harmonic photons from the fundamental light and any background signal.

The polymer particles (PSS) were spherical polystyrene microspheres (Polysciences) narrowly distributed about a 1.05 $\pm 0.03 \mu \mathrm{m}$ diameter. The particle surfaces are negatively charged owing to the presence of sulfate $\left(-\mathrm{SO}_{4}{ }^{-}\right)$groups. All PSS samples were prepared in doubly distilled water at a particle density of $1 \times 10^{8}$ particles $/ \mathrm{cm}^{3}$. The oil/water emulsion was prepared by mixing a $5 \mathrm{mM} 1$-dodecanol (Aldrich) $/ n$-tetradecane (Aldrich) solution with a $5 \mathrm{mM}$ sodium dodecyl sulfate, SDS, (Sigma)/water solution at a volume/volume ratio 1:9. 1-Dodecanol is a coemulsifier in the emulsion. The average size of the emulsion was found to be $0.220 \pm 0.003 \mu \mathrm{m}$ by applying Mie scattering theory to the turbidity data. ${ }^{24-25}$ The emulsion was diluted to a droplet density of $6 \times 10^{10}$ droplets $/ \mathrm{cm}^{3}$. The turbidity of each sample was measured by a UV-vis spectrometer (Perkin-Elmer, Lambda 19). The stabilities of the PSS suspensions and the emulsion at different concentrations of electrolyte solution were checked. The UV-visible spectra of all the samples for both PSS and emulsion systems did not change upon addition of electrolyte and changed less than $3 \%$ before and after the SHG measurement.

Potassium chloride, sodium bromide, and magnesium sulfate (Aldrich) were heated to $600{ }^{\circ} \mathrm{C}$ for more than $8 \mathrm{~h}$ before use. All salt solutions were prepared in doubly distilled water. The solution $\mathrm{pH}$ was $6.3 \pm 0.2$. All measurements were performed at $22{ }^{\circ} \mathrm{C}$.

\section{Results and Discussion}

Although the Gouy-Chapman model is derived for charged planar interfaces, it is applicable to the charged curved surfaces of the PSS and emulsion systems used in the present studies. It is known that a charged curved surface in contact with an electrolyte solution can be approximated by a flat surface when the product of the inverse of the Debye length, $\kappa$, and the radius of the particles, $r$, is large, $\kappa r>15 .{ }^{26}$ With this approximation the Poisson-Boltzmann equation can be solved as if the surface is planar, which is the Gouy-Chapman model, i.e., eq 7. In the intermediate regime $1<\kappa r<15$, the approximate solution of the Poisson-Boltzmann equation for the potential profile around a spherical colloidal particle is needed. For the PSS system, the range is $465>\kappa r>51$, and therefore it is valid to apply the Gouy-Chapman model to describe the potential of the PSS particle. Although $\kappa r$ ranges from 5 to 60 in the emulsion studies, it was found that the deviation of the charge density calculated from the zeroth order approximate solution of the Poisson-Boltzmann equation relative to the exact numerical solution of the Gouy-Chapman model is less than $10 \%$ for $\kappa r=5 .{ }^{26}$ When the charge density differs by $10 \%$ at $\kappa r=5$, the corresponding $\Phi(0)$ 's calculated by the GouyChapman model differs by $4 \%$, which is within the experimental error of the SHG measurements.

The second harmonic intensity, which is the square of the SH field, was measured as functions of $[\mathrm{KCl}]$ and $\left[\mathrm{MgSO}_{4}\right]$ for the PSS system and as functions of $[\mathrm{NaBr}]$ and $\left[\mathrm{MgSO}_{4}\right]$ for the emulsion system. The SHG data for the different electrolyte concentrations were fitted to eq 8 . From the fitting $A, B$, and $\sigma$ were obtained. $\Phi(0)$ could then be found for a given 

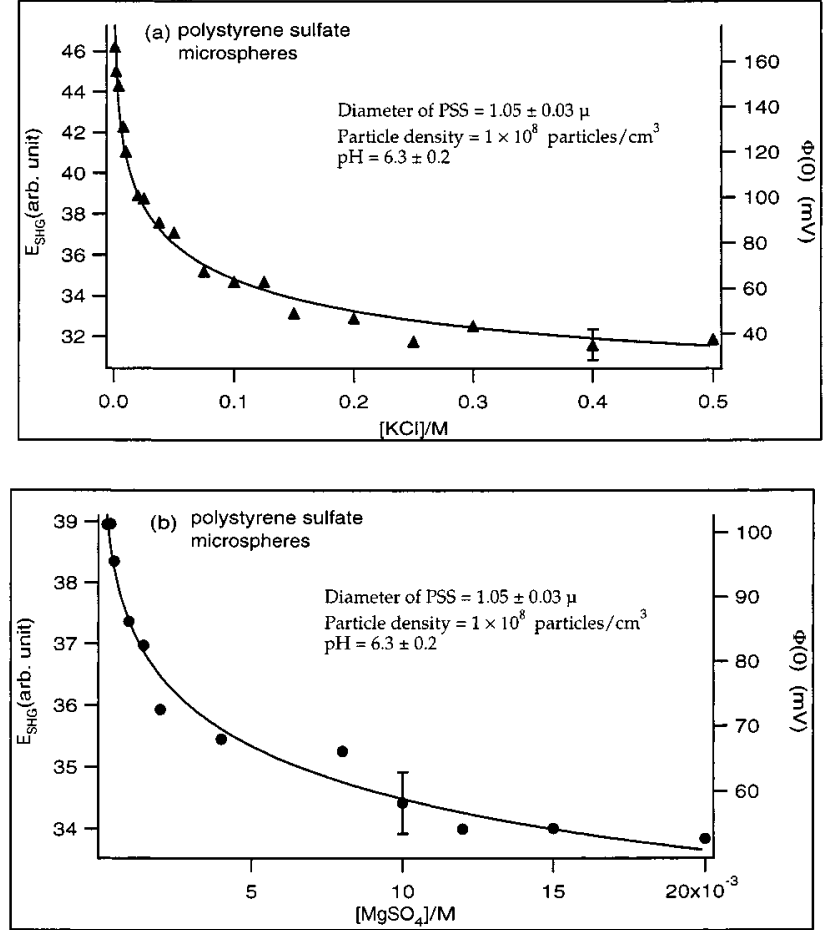

Figure 2. Second harmonic field, $E_{\mathrm{SHG}}$, and surface potential, $\Phi(0)$, plotted as a function of (a) $[\mathrm{KCl}]$ and (b) $\left[\mathrm{MgSO}_{4}\right]$ for the polystyrene sulfate microsphere (PSS) system. The triangles and circles are the experimental data. The solid curves are obtained by using the GouyChapman model to fit the experimentally determined $E_{\mathrm{SHG}}$, eq 8.

electrolyte solution using eq 6 . It was found that the best fits of $A, B$, and $\sigma$ were obtained by using data from both univalent (1:1) electrolyte and divalent (2:2) electrolyte experiments.

The second harmonic field, $E_{\mathrm{SHG}}$, is plotted as a function of $[\mathrm{KCl}]$ and $\left[\mathrm{MgSO}_{4}\right]$ for the PSS system, Figure 2, and as a function of $[\mathrm{NaBr}]$ and $\left[\mathrm{MgSO}_{4}\right]$ for the emulsion system, Figure 3. The three fitting parameters $A, B$, and $\sigma$ found from the fitting are shown in Table 1 for the PSS system and Table 2 for the emulsion system. They show that both independent sets of data for $\mathrm{KCl}$ and $\mathrm{MgSO}_{4}$ are in agreement for the PSS, and the data for $\mathrm{NaBr}$ and $\mathrm{MgSO}_{4}$ are in agreement for the emulsion system. With $A$ and $B$ known from the fitting and $E_{\mathrm{SHG}}$ measured experimentally, the surface potential, $\Phi(0)$, can be found by solving eq 6 . With the surface charge density, $\sigma$, obtained from the fitting, $\Phi(0)$ can be obtained by the Gouy-Chapman model; see eq 7. Figures 2 and 3 show the experimentally determined $\Phi(0)$ and the theoretical fit of Gouy-Chapman model.

A further demonstration that the Gouy-Chapman model contained in the expression for the $\mathrm{SHG}$, eq 8, is applicable to the PSS and emulsion studies is the dependence of the SH signal on the valence, $Z$, of the electrolyte. The $Z$ dependence is explicitly shown in eq 8 . A convenient way to examine this dependence of $E_{\mathrm{SHG}}$ on $Z$ is to use the approximation, $\sinh ^{-1} x$ $\approx \ln 2 x$ for $x>3$. This approximation is valid at electrolyte concentrations below $10^{-2} \mathrm{M}$. Using it makes it possible to separate the electrolyte concentration, $C$, and the charge density, $\sigma$, in the expression for the second harmonic field, $E_{\mathrm{SHG}}$, as follows

$$
E_{\mathrm{SHG}}=A+B\left(\frac{2 k T}{Z e}\right) \ln \left(2 \sigma \sqrt{\frac{\pi}{2 k T \epsilon}}\right)-B\left(\frac{k T}{Z e}\right) \ln C
$$

In this form if the experimentally determined value for $E_{\mathrm{SHG}}$ is plotted against the $\ln C$, a straight line should be obtained.
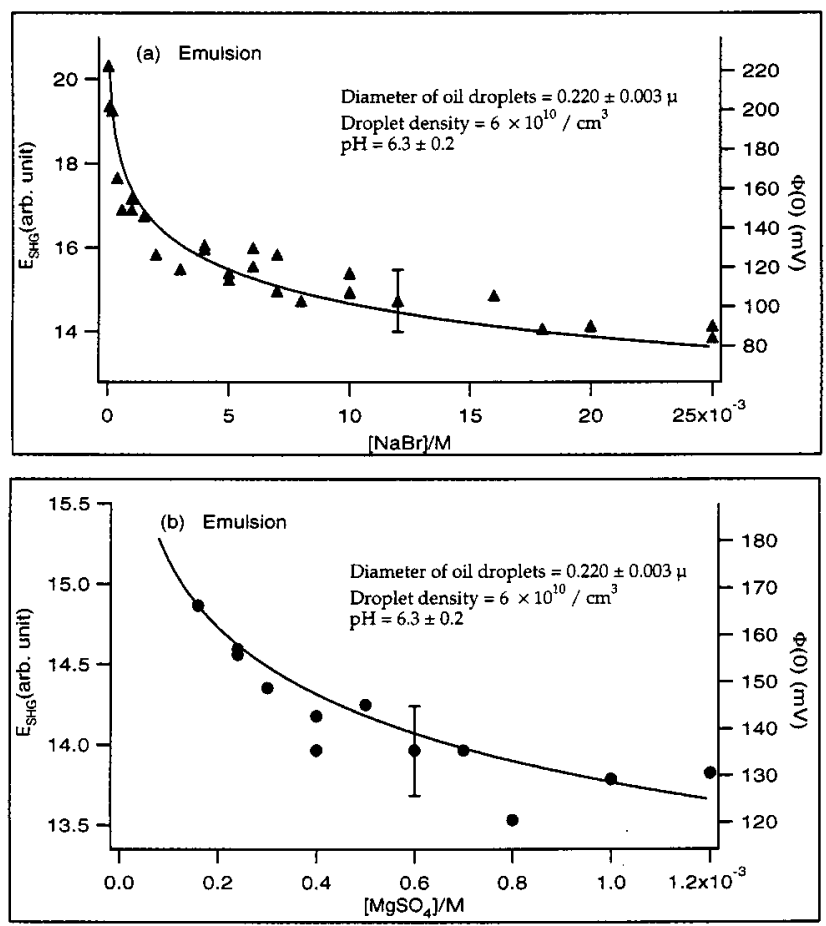

Figure 3. Second harmonic field, $E_{\mathrm{SHG}}$, and surface potential, $\Phi(0)$, plotted as a function of (a) $[\mathrm{NaBr}]$ and (b) $\left[\mathrm{MgSO}_{4}\right]$ for the emulsion system. The triangles and circles are the experimental data. The solid curves are obtained by using the Gouy-Chapman model to fit the experimentally determined $E_{\mathrm{SHG}}$, eq 8 .

TABLE 1: Results of the Fitting for $E_{\mathrm{SHG}}$ vs $C$ by Eq 8 and $E_{\text {SHG }}$ vs ln $\mathrm{C}$ by $\mathrm{Eq} 9$ for $\mathrm{KCl}$ and $\mathrm{MgSO}_{4}$ for the Polystyrene Sulfate Microspheres (PSS) in Water

\begin{tabular}{llll}
\hline & & \multicolumn{2}{c}{ polystyrene sulfate microspheres } \\
\cline { 3 - 4 } & & \multicolumn{1}{c}{$\mathrm{KCl}$} & \multicolumn{1}{c}{$\mathrm{MgSO}_{4}$} \\
\hline$E_{\text {SHG vs C }}$ & $A$ & $28 \pm 0.7$ & $29 \pm 0.7$ \\
& $\sigma\left(\right.$ charges $\left./ \mathrm{cm}^{2}\right)$ & $(3.7 \pm 0.3) \times 10^{13}$ & $(3.6 \pm 0.5) \times 10^{13}$ \\
& $B(2 k T / Z e)$ & $5.6 \pm 0.2$ & $2.5 \pm 0.1$ \\
$E_{\text {SHG vs ln C }}$ & slope $=-B(k T / Z e)$ & $2.5 \pm 0.2$ & $1.2 \pm 0.1$
\end{tabular}

TABLE 2: Results of the Fitting for $E_{\mathrm{SHG}}$ vs $C$ by Eq 8 and $E_{\mathrm{SHG}}$ vs $\ln C$ by $\mathrm{Eq} 9$ for $\mathrm{NaBr}$ and $\mathrm{MgSO}_{4}$ for the Tetradecane Oil in Water Emulsion

\begin{tabular}{llcc}
\hline & & \multicolumn{2}{c}{ emulsion } \\
\cline { 3 - 4 } & & $\mathrm{NaBr}$ & $\mathrm{MgSO}_{4}$ \\
\hline$E_{\text {SHG vs } C}$ & $A$ & $9.9 \pm 0.3$ & $10.0 \pm 0.3$ \\
& $\sigma\left(\right.$ charges $\left./ \mathrm{cm}^{2}\right)$ & $(3.3 \pm 0.5) \times 10^{13}$ & $(3.3 \pm 0.5) \times 10^{13}$ \\
& $B(2 k T / Z e)$ & $2.2 \pm 0.2$ & $1.15 \pm 0.05$ \\
$E_{\text {SHG vs } \ln C}$ & slope $=-B(k T / Z e)$ & $1.2 \pm 0.1$ & $0.59 \pm 0.08$
\end{tabular}

Furthermore, the slope of the line would be inversely proportional to the electrolyte valence $Z$. Thus the slope for the univalent electrolytes $\mathrm{KCl}$ and $\mathrm{NaBr}$ should be twice the slope for $\mathrm{MgSO}_{4}$. Figure 4 shows the plots of $E_{\mathrm{SHG}} \mathrm{vs} \ln [\mathrm{KCl}]$ and $E_{\mathrm{SHG}} \mathrm{vs} \ln \left[\mathrm{MgSO}_{4}\right]$ for the PSS system. Two straight lines are obtained. The slope for $\mathrm{KCl}$ is equal to $-2.5 \pm 0.2$ and the slope for $\mathrm{MgSO}_{4}$ is equal to $-1.2 \pm 0.1$, yielding a ratio of 2.1:1, which shows very good agreement with the theoretical prediction of 2:1. Good agreement was also found for the emulsion system; see Figure 5 and Table 2. The slope for $\mathrm{NaBr}$ is equal to $-1.2 \pm 0.1$ and the slope for $\mathrm{MgSO}_{4}$ is equal to $-0.59 \pm 0.08$, yielding a ratio of $2.0: 1$, versus the theoretical prediction of $2: 1$.

It is to be noted that we obtain the absolute value of the surface potential of a microparticle by the SHG method. There is no need for a calibration because the actual surface charge 


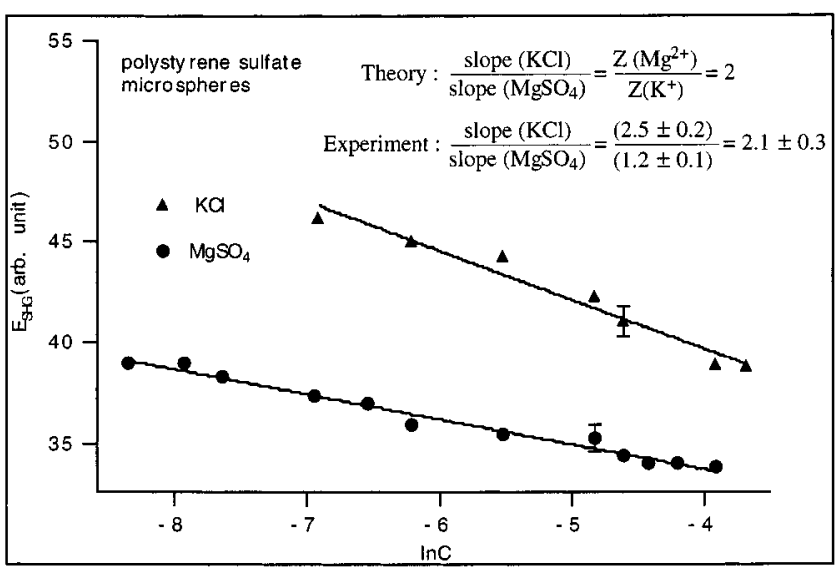

Figure 4. Dependence of the second harmonic field, $E_{\mathrm{SHG}}$, on the electrolyte valences, $1: 1$ and 2:2. The $E_{\mathrm{SHG}}$ is plotted as a function of $\ln [\mathrm{KCl}]$ and $\ln \left[\mathrm{MgSO}_{4}\right]$ for the polystyrene sulfate system (PSS) system. The triangles and circles are the experimental values. The solid lines are obtained by fitting the data to the Gouy-Chapman model, eq 9.

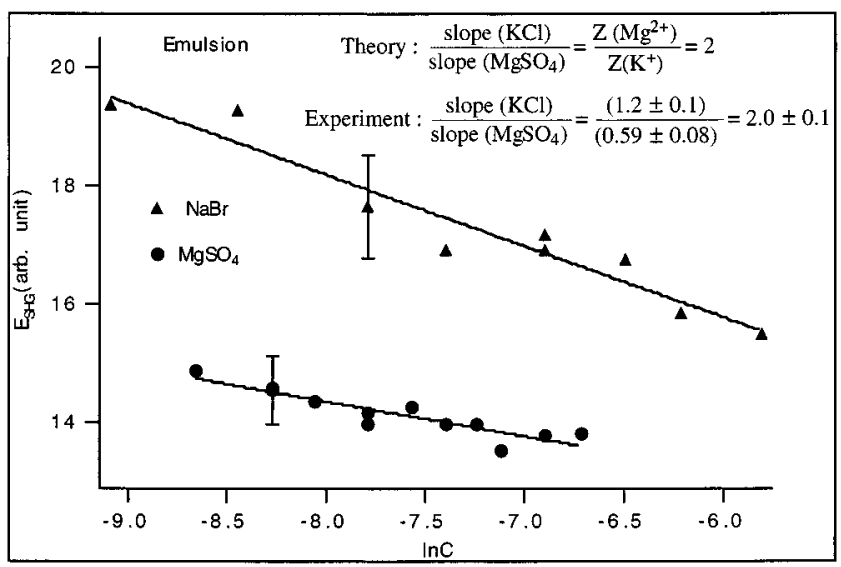

Figure 5. Dependence of the second harmonic field, $E_{\mathrm{SHG}}$, on the electrolyte valences, $1: 1$ and $2: 2$. The $E_{\mathrm{SHG}}$ is plotted as a function of $\ln [\mathrm{NaBr}]$ and $\ln \left[\mathrm{MgSO}_{4}\right]$ for the emulsion system. The triangles and circles are the experimental values. The solid line are obtained by fitting the data to the Gouy-Chapman model, eq 9.

density is obtained from fitting the experimental data to eq 8 . The surface potential can then be found from the GouyChapman model, eq 7, at a given electrolyte concentration and the experimentally obtained surface charge density, $\sigma$.

It is useful to compare the $\Phi(0)$ obtained from SHG measurements, $\Phi(0)=\left(E_{\mathrm{SHG}}-A\right) / B$, with the zeta potential, $\zeta$, obtained from electrophoresis experiments. The $\zeta$ 's measured for the PSS system at $[\mathrm{KCl}]=0.01$ and $0.1 \mathrm{M}$ are $-73 \pm 5$ and $-37 \pm 3 \mathrm{mV}$ respectively, while $\Phi(0)$ obtained from SHG are $-120 \pm 11$ and $-62 \pm 10 \mathrm{mV}$, respectively. It is to be noted that $\Phi(0)$ measured by SHG is larger than $\zeta$, which is consistent with the fact that $\zeta$ is the potential at the shear surface, which is located further away from the surface charges.

The surface potential $\Phi(0)$, which is obtained by the SHG method, is the potential drop across a Gouy-Chapman diffuse double layer with the potential of the bulk liquid far from the microparticle surface being set equal to zero. A theoretical relation between $\Phi(0)$ and $\zeta$ has been established based on a model in which the shear surface is replaced by a shear zone. The viscosity in the shear zone changes continuously rather than discontinuously, i.e., with an infinite jump in viscosity ${ }^{27,28}$ for the case of a sharp shear surface. The potential drop across the Gouy-Chapman diffuse double layer, $\Phi(0)$, is evaluated as the potential at the inner surface of this shear zone. For a concentration of $1: 1$ electrolyte at $0.1 \mathrm{M}$ and a $\Phi(0)$ of -62 $\mathrm{mV}$ obtained from $\mathrm{SHG}$, the theoretical model predicts a zeta potential $\xi$ of $-35 \mathrm{mV}$. The electrophoresis measurement yields a $\zeta$ of $-37 \mathrm{mV}$. For a concentration of $1: 1$ electrolyte at 0.01 $\mathrm{M}$ and a $\Phi(0)$ of $-120 \mathrm{mV}$ obtained from SHG, the theoretical model predicts a $\zeta$ of $-75 \mathrm{mV}$. The electrophoresis measurement gives a $\xi$ of $-73 \mathrm{mV}$. We thus observe that the theoretical model relating the surface potential, $\Phi(0)$, and the zeta potential, $\zeta$, is in remarkably good agreement with the values of $\Phi(0)$ obtained from SHG and $\zeta$ obtained from electrophoresis.

\section{Conclusion}

We have used a second harmonic method to investigate the electrostatic properties of microscopic particle surfaces. This noninvasive method was used to study the aqueous/polystyrene sulfate microsphere interface and the aqueous/oil (tetradecane) droplet emulsion interface. The dependences of the second harmonic signal on electrolyte concentration in the bulk solution, and on the valences of the electrolyte, i.e., univalent $\mathrm{K}^{+}, \mathrm{Na}^{+}$ and divalent $\mathrm{Mg}^{2+}$, are in excellent agreement with the GouyChapman model. From the fitting of the second harmonic measurements to the Gouy-Chapman model, we have obtained the surface charge density and the surface potential of the microscopic particles. The comparison of the surface potential, $\Phi(0)$, obtained from SHG and the zeta potential, $\zeta$, obtained by electrophoresis is found to be in excellent agreement with theoretical predictions based on a model in which the surface potential is considered as the inner surface of the shear zone while the zeta potential is considered as the outer surface of the shear zone.

Acknowledgment. The authors thank the Division of Chemical Science of the Department of Energy for their support and the National Science Foundation for their equipment support. The contribution from Dr. D. Zimdars and Dr. H. Wang to this work is gratefully acknowledged.

\section{References and Notes}

(1) Hiemenz, P. C. Principles of colloid and surface chemistry, 2nd ed.; Marcel Dekker: New York, 1986.

(2) Fitch, R. M. Polymer Colloids - A Comprehensive Introduction; Academic Press: New York, 1997.

(3) Sprycha, R.; Matijevic, E. Langmuir 1989, 5, 479.

(4) McLaughlin, S.; Whitaker, M. J. Physiol. 1988, 396, 189.

(5) Ermakov, Y. A. Biochim. Biophys. Acta. 1990, 1023, 91. 5,1258

(6) Kandori, K.; Ishiguto, H.; Kon-no, K.; Kitahara, A. Langmuir 1989,

(7) Barchini, R.; Saville, D. A. Langmuir 1996, 12, 1442.

(8) Abuin, E. B.; Lissi, E. A.; Núñez, R.; Olea, A. Langmuir 1989, 5 , 753. 74.

(9) Hobson, R. A.; Grieser, F.; Healy, T. W. J. Phys. Chem. 1994, 98,

(10) Malyarenko, V. V.; P. Kuprienko, I. Colloid J. USSR 1995, 57, 198.

(11) Takeoka, S.; Ohgushi, T.; Terase, K.; Ohmori, T.; Tuschida, E. Langmuir 1996, 12, 1766.

(12) Larson, I.; Drummond, C. J.; Chan, D. Y. C.; Grieser, F. J. Am. Chem. Soc. 1993, 115, 11885.

(13) Richmond, G.; Robinson, J. M.; Shannon, V. L. Prog. Surf. Sci. 1988, 28,1 .

(14) Shen, Y. R. Апnи. Rev. Phys. Chem. 1989, 40, 327.

(15) Corn, R. M.; Higgins, D. A. Chem. Rev. 1994, 94, 107.

(16) Eisenthal, K. B. Chem. Rev. 1996, 96, 1343.

(17) Wang, H.; Yan, E. C. Y.; Borguet, E.; Eisenthal, K. B. Chem. Phys. Lett. 1996, 259, 15.

(18) Wang, H.; Yan, E. C. Y.; Liu, Y.; Eisenthal, K. B. J. Phys. Chem $B$ 1998, 102, 4446 . 327. 
(20) Zhao, X.; Ong, S.; Eisenthal, K. B. Chem. Phy. Lett. 1993, 202, 513.

(21) Zhao, X.; Ong, S.; Wang, H.; Eisenthal, K. B. Chem. Phy. Lett. 1993, 214, 203.

(22) Terhune, R. W.; Maker, P. D.; Savage, C. M. Phys. Rev. Lett. 1963, 14,681

(23) Clays, K.; Persoons, A. Phys. Rev. Lett. 1991, 66, 2980.
(24) Kerker, M. Electromagnetic Scattering Proceedings; Macmillan: New York, 1963; pp 122-131.

(25) Tabibian, R.; Heller, W.; Epel, J. N. J. Colloid Sci. 1956, 11, 195.

(26) White, L. R. J. Chem. Soc., Faraday Trans. 2 1977, 73, 577.

(27) Lyklema, J.; Oberbeek, J. J. Colloid Sci 1961, 16, 501.

(28) Hiemenz, P. C. Principles of colloid and surface chemistry, 2nd ed.; Marcel Dekker: New York, 1986; Chapter 13. 